\title{
"Leaving nothing behind": is the bioresorbable vascular scaffold a new hope for patients with coronary artery disease?
}

\author{
Maciej Lesiak, Aleksander Araszkiewicz \\ $1^{\text {st }}$ Department of Cardiology, Poznan University of Medical Sciences, Poznan, Poland
}

Postep Kardiol Inter 2014; 10, 4 (38): 283-288

DOI: $10.5114 /$ pwki.2014.46940

\begin{abstract}
A bstract
Despite significant advances in design and technology of drug eluting stents (DES) and improved long-term outcome of patients treated with percutaneous coronary intervention, the implantation of metallic stents is associated with some limitations. Multiple stents, covering long coronary segments substantially affect vasomotion, changing the vessel into a rigid tube. Bioresorbable vascular scaffolds (BVS) promise complete bioresorption after 2 to 3 years, vessel lumen enlargement, reduction of the plaque to media ratio, and restoration of vasomotion. Thus BVS seems to be a new, promising, and perhaps even a breakthrough invasive treatment for patients with coronary artery disease. The results of randomised trials and registries confirm the efficacy and safety of the BVS, provided the compliance with the technical aspects of implantation. A key role plays also the selection of patients who could potentially benefit most from the implantation of the BVS. The idea of "leaving nothing behind" after percutaneous coronary interventions is a very exiting concept in modern interventional cardiology. If current technology meets the challenge, major limitations will be overcome, and scaffolds prove to be at least as safe and effective as current DES, than in a long run we will be facing a real breakthrough not only in cardiology, but generally in medicine.
\end{abstract}

Key words: bioresorbable vascular scaffold, coronary angioplasty.

\section{Introduction}

Despite significant advances in design and technology of drug-eluting stents (DES) and improved long-term outcome of patients treated with percutaneous coronary angioplasty, the implantation of metallic stents is associated with some limitations. Multiple stents, covering long coronary segments, substantially affect vasomotion, changing the vessel into a rigid tube. Prolonged contact with a foreign material, either a metal alloy or more often a polymer, stimulates inflammatory and thrombotic reactions and accelerates neoatherosclerosis, which increases the risk of stent thrombosis, myocardial infarction and repeat interventions [1, 2]. Although the majority of these adverse effects were reported in relation to the first generation DES, also modern stents, by trapping the coronary artery in a metallic cage, disturb physiological blood flow, hinder the non-invasive assessment of stented segments and exclude the possibility of future graft anastomosis in case of the need for bypass surgery (CABG).

For this reason, the bioresorbable vascular scaffold (BVS) may become a very promising alternative to DES.
After implantation, the scaffold maintains sufficient radial strength to prevent vessel recoil and releases the drug to inhibit the proliferation of neointima. Over time, the scaffold mass and its radial strength gradually decrease, and after 2-3 years the device vanishes completely, leaving the vessel covered with a healthy endothelium and normal vasomotor function. There is a growing body of long-term outcome data available suggesting the safety and efficacy of this novel technology.

\section{Device description}

Currently, the only BVS available in Poland is the Absorb BVS (Abbott Vascular, Santa Clara, CA, USA). This fully bioresorbable scaffold consists of a polymer backbone composed of poly L-lactide acid (PLLA), covered by an amorphous matrix, a 1:1 mixture of everolimus and another polymer, poly-DL-lactide (PDLLA). The scaffold has a corrugated ring design and is mounted on a Xience delivery catheter. Since the scaffold itself is radio-transparent, the 2 platinum markers are placed close to the proximal and distal edge of the device to allow its proper

\section{Corresponding author:}

Aleksander Araszkiewicz MD, PhD, $1^{\text {st }}$ Department of Cardiology, University of Medical Sciences, 1/2 Dluga St, 61-848 Poznan, Poland, phone: +48 608574 375, fax: +48 6185490 94, e-mail: aaraszkiewicz@interia.pl

Received: 11.11.2014, accepted: 12.11.2014. 
positioning. The resorption process progresses gradually, mainly due to hydrolysis; thus minimal or no inflammation can be observed [3]. The final products of this process are $\mathrm{CO}_{2}$ and $\mathrm{H}_{2} \mathrm{O}$. The scaffold is covered with everolimus, a potent antiproliferative drug, which is released at the same rate and amount as from metallic Xience $\mathrm{V}$. The Absorb BVS is a thick-strut scaffold, with the average strut thickness of 157 microns. Currently 3 diameters $(2.5,3.0,3.5 \mathrm{~mm})$, and 5 lengths $(8,12,18,23,28 \mathrm{~mm})$ are available.

\section{Rationale and clinical indications}

There are numerous potential advantages of a BVS over a metallic DES. The liberation of the coronary artery from the permanent scaffold re-establishes physiological vasomotion, restores vessel original geometry and preserves laminar blood flow. This in turn may limit the development of neoatherosclerosis. Contrary to metallic stents, where late luminal loss deteriorates the early result of percutaneous coronary intervention $(\mathrm{PCI})$, after deployment of the BVS a late luminal gain has been observed [4]. The absence of permanent foreign material reduces the risk of late stent thrombosis, myocardial infarction, and sudden death. Preliminary data suggest that the longer the observation time, the greater the advantage of the BVS over metallic stents. Thus it is obvious that the BVS should be predominantly used in young patients, particularly with long lesions or diffuse disease where multiple long stents are required. Especially patients with lesions located in the mid portion of the left anterior descending coronary artery, obtuse marginal branch or distal right coronary artery will benefit because the use of the BVS will allow for potential future bypass grafting. Regardless of the lack of sufficient data, it seems that the use of the BVS should not be restricted to subjects with stable coronary artery disease, since patients with acute coronary syndromes, including ST-segment elevation myocardial infarction, will have the same benefit in the long run.

\section{Clinical studies}

Two designs of the BVS have been assessed in clinical trials. The safety and feasibility of the BVS 1.0 was tested in the open-label prospective ABSORB Cohort A trial - "A bioresorbable everolimus-eluting coronary stent system for patients with single de novo coronary artery lesions" [5]. At 6 months, the angiographic in-stent late lumen loss (LLL) was $0.44 \mathrm{~mm}$ with evidence of scaffold recoil $(-11.8 \%)$ as measured by intravascular ultrasound (IVUS) $[6,7]$. However, vasomotion appeared to be restored, with inducible vasoconstriction and vasodilatation possible in the treated segment [6]. To improve the mechanical characteristics of the BVS and reduce recoil, a second-generation BVS (1.1) has been introduced. The BVS 1.1 has a smaller maximum circular unsupported surface area, a more uniform strut distribution, and improved stent retention [8]. The efficacy of the BVS 1.1 was assessed in the ABSORB Cohort B trial, which recruited 101 patients with single or 2-vessel de novo disease, with all receiving a $3 \times 18 \mathrm{~mm}$ BVS [9]. At 6-month follow-up, there was only 1 case of target vessel revascularization (TVR), while LLL was only $0.19 \pm 0.18 \mathrm{~mm}$; at 2-year follow-up, LLL was $0.27 \pm 0.20 \mathrm{~mm}$. Although after 6 months there was a significant reduction in minimal lumen area (MLA) on IVUS, as compared with the baseline $\left(6.60 \pm 1.22 \mathrm{~mm}^{2}\right.$ to $\left.6.37 \pm 1.12 \mathrm{~mm}^{2}, p<0.005\right)$, the scaffold area gradually increased with longer observation time $[9,10]$. Multimodality imaging observations of the ABSORB Cohort B patients after 3 years have been recently published [10]. On IVUS, mean lumen and scaffold area remained stable between the $2^{\text {nd }}$ and $3^{\text {rd }}$ year, whereas a significant reduction in plaque area behind the struts was observed with a trend toward adaptive restrictive remodelling of the external elastic membrane. Hyperechogenicity of the vessel wall, a surrogate of the bioresorption process, decreased from $23.1 \%$ to $10.4 \%$, with a reduction of radiofrequency backscattering for dense calcium and the necrotic core. The count of strut cores detected on optical coherence tomography (OCT) increased significantly, most likely reflecting the dismantling of the scaffold. Importantly, $98 \%$ of struts were covered with endothelium. At 3-year follow-up, there were $7(7 \%)$ ischaemia-driven target lesion revascularisations (TLR) and 3 (3\%) non-ST segment elevation myocardial infarctions (MI). The major adverse cardiovascular event (MACE) rate was $10.0 \%$, without any scaffold thrombosis.

Following this trial, ABSORB EXTEND, a prospective, single-arm, open-label clinical registry, was initiated as a global continued access study to expand the experience with the Absorb BVS system to different geographies (80 centres worldwide), with broader inclusion criteria to include the treatment of longer lesions and multiple vessels [11]. Patients with lesions $\leq 28 \mathrm{~mm}$ in length and reference vessel diameter of $2.0-3.8 \mathrm{~mm}$ have been included. At 1 year, for the first 512 patients enrolled in the study, the composite endpoints of ischaemia-driven MACE and target vessel failure were $4.3 \%$ and $4.9 \%$, respectively. The cumulative rate of Academic Research Consortium (ARC) defined definite and probable scaffold thrombosis for this population was $0.8 \%$ at 1 year [12].

The ABSORB II trial was designed to compare an everolimus-eluting bioresorbable scaffold with an everolimus-eluting metallic stent (Xience) [13]. In this single-blind, multicentre, randomised trial, 501 patients with evidence of myocardial ischaemia and 1 or 2 de-novo native lesions in different epicardial vessels were enrolled. The patients were randomized in a $2: 1$ ratio to receive treatment with a BVS or treatment with an everolimus-eluting metallic stent. The co-primary endpoints of this study were vasomotion (change in mean lumen diameter before and after nitrate administration 
at 3 years) and the difference between minimum lumen diameter (after nitrate administration) after the index procedure and after 3 years. Secondary endpoints were: procedural performance of the device, assessed by quantitative angiography and intravascular ultrasound; composite clinical endpoints (death, myocardial infarction, and coronary revascularization); device and procedural success; and angina status assessed by the Seattle Angina Questionnaire (SAQ) and exercise testing at 6 and 12 months. The 1-year results of ABSORB II were recently published in The Lancet [13]. Cumulative rates of first new or worsening angina, from adverse event reporting, were lower (72 patients [22\%] in the bioresorbable scaffold group vs. 50 [30\%] in the metallic stent group, $p=$ 0.04). Patients' performance during maximum exercise and angina status measured by SAQ were similar. The 1-year composite, device-orientated endpoint was similar for BVS and DES groups (16 patients [5\%] vs. 5 patients [3\%], $p=0.35$ ). Three patients in the BVS group developed definite or probable scaffold thrombosis (1 definite acute, 1 definite sub-acute, and 1 probable late), as compared with no patients in the metallic stent group. There were 17 cases of MACE (5\%) in the BVS group, as compared with 5 (3\%) events in the DES group. The most common adverse events were MI (15 cases [4\%] vs. 2 cases [1\%], respectively) and clinically indicated TLR ( 4 cases [1\%] vs. 3 cases [2\%], respectively).

In conclusion the authors stated that the BVS showed a similar 1-year clinical outcome to the everolimus-eluting metallic stent.

Conversely, heterogeneous outcomes have been reported from registries enrolling a more complex population, as compared with the ABSORB II study. The results of the GHOST registry were published recently [14]. A total of 1189 patients underwent $\mathrm{PCI}$ with the implantation of 1 or more BVS in 10 high-volume European centres. In the registry patients with acute coronary syndromes, calcified lesions, chronic total occlusions (СTO), or complex bifurcation lesions were included. The primary outcome was target lesion failure (TLF), defined as the combination of cardiac death, target vessel myocardial infarction, or clinically driven TLR. A total of 1731 BVS were implanted. Technical success was achieved in $99.7 \%$ of cases. TLF occurred in 67 out of 1189 patients at a median of 109 (interquartile range: 8-227) days after implantation. The cumulative incidence of TLF was $2.2 \%$ at 30 days and $4.4 \%$ at 6 months. The annualised rate of TLF was $10.1 \%$. At 6 months, the rate of cardiac death was $1.0 \%$, target vessel myocardial infarction $2.0 \%$, TLR $2.5 \%$, and TVR $4.0 \%$. The cumulative incidence of definite/ probable scaffold thrombosis was $1.5 \%$ at 30 days and $2.1 \%$ at 6 months, with 16 of 23 cases occurring within 30 days after index $\mathrm{PCl}$. Real-world outcomes of the BVS in the GHOST registry showed acceptable rates of TLF at 6 months, although the rates of early and midterm scaf- fold thrombosis, mostly clustered within 30 days, were not negligible.

The recently published BVS STEMI first study is a prospective, single-arm, single-centre study, reporting data following BVS implantation in 49 STEMI patients [15]. The procedural success was $97.9 \%$, while no patients had angiographically visible residual thrombus at the end of the procedure. The OCT analysis (performed in 31 patients) showed that the mean percentage of malapposed struts per patient was $2.80 \pm 3.90 \%$. At 30-day follow-up, the TLF rate was $0 \%$ and no death or scaffold thrombosis was reported. Other interesting results in the STEMI subset come from the Prague-19 multicentre study, where 40 patients undergoing primary $\mathrm{PCl}$ were evaluated. The 6-month survival free from death, $\mathrm{MI}$ or TVR was $95 \%$, while an OCT substudy (performed on 21 patients) demonstrated that only $1.1 \%$ of scaffold struts were malapposed [16].

\section{Scaffold implantation technique}

Due to a different structure and mechanical properties of the BVS as compared with metallic stents, some technical issues regarding implantation technique need to be discussed. The polymeric nature of the scaffold carries physical limitations that should be taken into account before deployment. Due to strict dilatation limits, overexpansion can lead to strut fracture or disruption of the scaffold inside the vessel. Careful vessel sizing before BVS implantation is crucial for procedural success. As a general rule, the BVS should not be implanted into lesions that cannot be adequately prepared with balloon inflations, particularly when the balloon used for pre-dilatation cannot be fully expanded or when the result of preparation is unsatisfactory. The deployment should proceed gradually, pressurizing the delivery system in $2 \mathrm{~atm}$ increments, every $5 \mathrm{~s}$ until complete expansion of the scaffold. Upon device deployment, target pressure should be maintained for at least $30 \mathrm{~s}$. Post-dilatation with larger balloons is possible, as long as overexpansion is not greater than $0.5 \mathrm{~mm}$, as compared with the scaffold nominal diameter. Upon deployment, one should aim to obtain $<10 \%$ residual stenosis, full scaffold expansion and optimal strut apposition. Therefore, post-dilatation with a high-pressure non-compliant balloon is advisable unless intracoronary imaging confirms full expansion and apposition. The proper technique of implantation allows one to avoid the possible complications including acute and subacute stent thrombosis.

\section{Special lesion subsets}

Although the majority of studies on the BVS enrolled patients with relatively simple lesions, it seems that some advantages should be expected also in more complex anatomy. Main branch stenting in a bifurcation lesion will prevent prolonged side branch jailing if a BVS 
is used. In ostial and aorto-ostial lesions some overhang of a scaffold will not produce permanent flow turbulence, nor will it cause any problem with future access to the vessel. In-stent restenosis also may become a potential indication for the use of scaffolds. Although vessel vasomotion will not be restored, the BVS will provide longer and more potent drug delivery as compared to drug-eluting balloons. Unlike DES, complete resorption of a scaffold will prevent long-term multiple metallic strut layering in a vessel lumen. Severely calcified vessels will not benefit from scaffold disappearance, because a positive effect of vasomotion will not appear anyway. Nevertheless, accurate lesion preparation, with optimal pre-dilatation or the use of additional devices such as cutting or scoring balloons or even rotational atherectomy, will make scaffold delivery and implantation possible in the majority of cases. Even such vessels will benefit from struts' disappearance by reduction of the risk of late stent thrombosis and possible late lumen gain.

When treatment of bifurcation lesions is planned with the BVS, the provisional stenting technique seems to be a logical approach. However, the BVS is a breakable device, so the proximal optimization technique, side branch (SB) fenestration, and kissing balloon inflation should be performed with caution. The bench tests showed that crossing the struts of the scaffold with a $2.5 \mathrm{~mm}$ balloon at a pressure of up to $8 \mathrm{~atm}$ can at most lead to the rupture of single struts, usually connectors. Typical kissing, due to the size of 2 balloons filled with high pressure, can damage the BVS (Figure 1). The authors of this review are conducting a registry on the use of the BVS in bifurcation lesions. Preliminary data were presented during the $10^{\text {th }}$ European Bifurcation Club meeting in Bordeaux this year [17]. So far, a total of 65 patients have reached a 9-month follow up. In the majority of cases a simple, single-stent strategy was used (85\%). In lesions where side branch stenting was required, only T or TAP techniques were used (Figure 2). Pre- and high pressure post-dilatations were performed in $98 \%$ and $75 \%$ respectively. Main vessel scaffolds were sized according to the proximal vessel reference diameter, and final kissing balloon post-dilatation with minimal protrusion of the side branch balloon (snuggle) was performed in 38\% of cases. After 9 months there was 1 case of cardiovascular death and 2 cases of subacute definite/probable scaffold prognosis. All patients remained on dual antiplatelet therapy for 12 months. There were no major ischaemic events between 30 days and long-term observation time. Both events of stent thrombosis occurred within the first 10 days after the procedure. This is why currently we recommend ticagrelor rather than clopidogrel at least for the first 3 months after the procedure.

Chronic total occlusion constitutes a special subset of coronary lesions, with the lowest $\mathrm{PCl}$ success rate irrespective of the approach. Long and diffuse disease, often with an extensive amount of calcium, as well as difficulty in the assessment of distal vessel diameter, makes the use of the BVS particularly challenging. However, adequate lesion preparation after wire crossing, nitroglycerine injection to dilate the vessel lumen and the use of intravascular imaging make BVS application possible. In a series of 40 CTO cases treated with BVS in the authors' institution, the acute results were satisfactory, with no in-hospital major complications. It should be noted that the majority of cases were quite simple lesions with the average J-CTO score of 1.7. In 2 patients retrograde technique was used, and the average number of scaffolds per patient was 1.6 (from 1 to 4 ). Since in many patients dissection-reentry technique was used, and because of the lack of adequate data, we avoided using the BVS to cover long subintimal channels. After 6 months of observation there were no deaths, and 1 case of scaffold thrombosis was observed with subsequent non ST-segment elevation myocardial infarction.
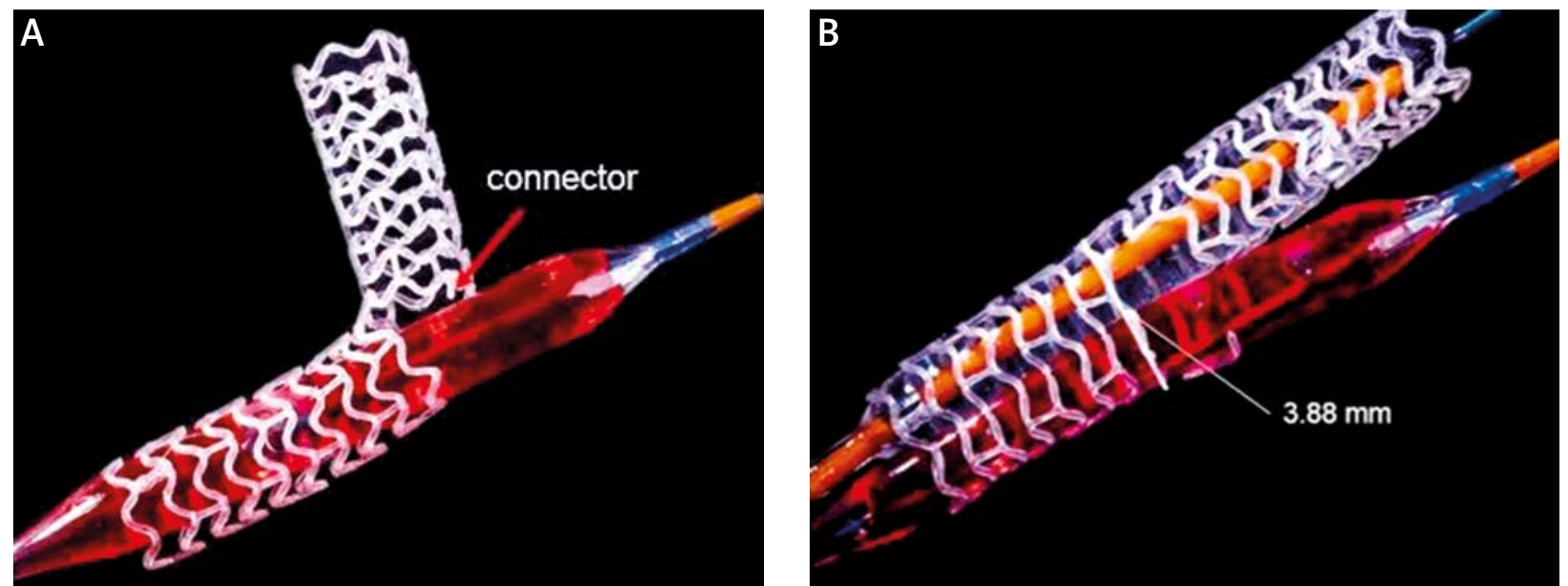

Figure 1. The bench tests of Absorb BVS. A - Single fracture of a connector of a $3.0 \times 18 \mathrm{~mm}$ scaffold after strut crossing with $2.5 \mathrm{~mm}$ balloon, at a pressure of $8 \mathrm{~atm}$. B - Major rupture of a $3.0 \times 18 \mathrm{~mm}$ scaffold after kissing balloon post-dilatation with 3.0 and 2.5 non-compliant balloons inflated up to $10 \mathrm{~atm}$ 

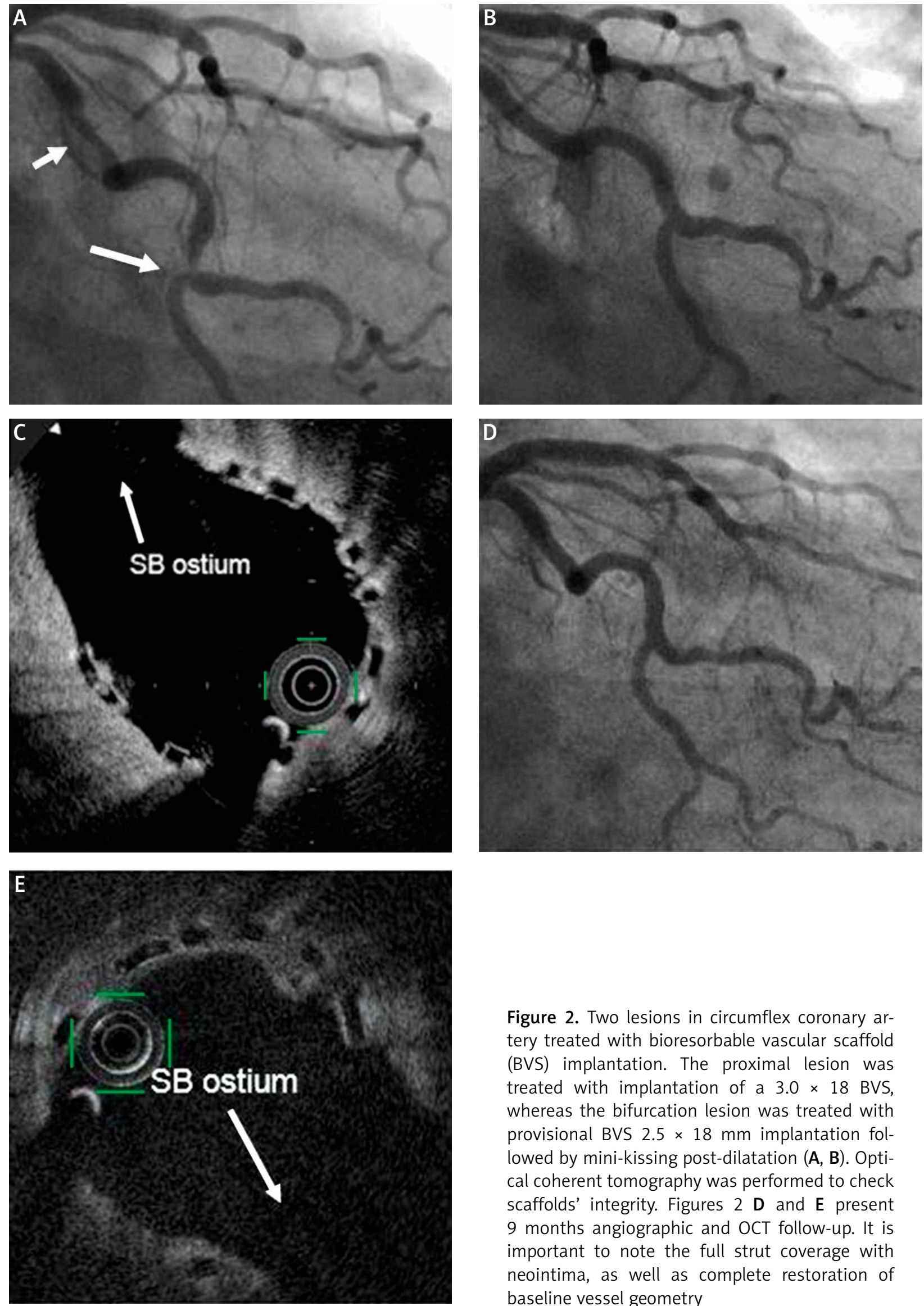

Figure 2. Two lesions in circumflex coronary artery treated with bioresorbable vascular scaffold (BVS) implantation. The proximal lesion was treated with implantation of a $3.0 \times 18$ BVS, whereas the bifurcation lesion was treated with provisional BVS $2.5 \times 18 \mathrm{~mm}$ implantation followed by mini-kissing post-dilatation (A, B). Optical coherent tomography was performed to check scaffolds' integrity. Figures 2 D and E present 9 months angiographic and OCT follow-up. It is important to note the full strut coverage with neointima, as well as complete restoration of baseline vessel geometry 


\section{Device limitations}

As with any novel technology, scaffolds have several limitations, many of them not negligible. The main limitations of the BVS are the thickness of struts and their potential for fracture. Thick struts make the whole device bulky (crossing profile $>1 \mathrm{~mm}$ ), hampering its passage across calcified bends or not optimally prepared lesions. For the same reason, use of $5 \mathrm{Fr}$ catheters, or support "mother-and-child" catheters is difficult. Additionally, thick-strut, as compared to thin-strut stents are more thrombogenic and less forgiving in relation to suboptimal deployment (malapposition, underexpansion, etc.) [18]. Polymeric scaffolds have restricted expansion limits. Post-dilatation with a balloon diameter more than $0.5 \mathrm{~mm}$ bigger than the scaffold diameter may lead to strut fracture with subsequent loss of radial strength and possible vessel collapse. Ruptured strut prolapse into the lumen of the vessel may disturb the flow, which increases the risk of thrombosis. The other limitations are: limited sizes and diameters currently available, the need for slow and prolonged dilatations (with the possibility of ischaemia), lack of visibility on X-ray imaging, and the need for longer and more potent dual antiplatelet therapy until specific data are available.

\section{Future directions}

The idea of "leaving nothing behind" after percutaneous coronary intervention is a very exciting concept in modern interventional cardiology. New generation metallic DES perform extremely well, being very effective and safe. However, after doing their great job, DES become redundant or even unwanted - as unwanted as a plaster cast on a leg after the bone is healed. If current technology meets the challenge, major limitations are overcome, and scaffolds prove to be at least as safe and effective as current DES, then in the long run we will face a real breakthrough not only in cardiology, but generally in medicine.

\section{References}

1. Lüscher TF, Steffel J, Eberli FR et al. Drug-eluting stent and coronary thrombosis. Biological mechanisms and clinical implications. Circulation 2007; 115: 1051-8.

2. Nakazawa G, Otsuka F, Nakano M, et al. The pathology of neoatherosclerosis in human coronary implants bare-metal and drug-eluting stents. J Am Coll Cardiol 2011; 57: 1314-22.

3. Otsuka F, Pacheco E, Perkins LE, et al. Long-term safety of an everolimus-eluting bioresorbable vascular scaffold and the cobalt-chromium XIENCE $V$ stent in a porcine coronary artery model. Circ Cardiovasc Interv 2014; 7: 330-42.

4. Serruys PW, Garcia-Garcia HM, Onuma Y. From metallic cages to transient bioresorbable scaffolds: change in paradigm of coronary revascularization in the upcoming decade? Eur Heart 2012; 33: 16-25.

5. Ormiston JA, Serruys PW, Regar E, et al. A bioabsorbable everolimus-eluting coronary stent system for patients with single de-novo coronary artery lesions (ABSORB): a prospective open-label trial. Lancet 2008; 371: 899-907.
6. Serruys PW, Ormiston JA, Onuma Y, et al. A bioabsorbable everolimus-eluting coronary stent system (ABSORB): 2-year outcomes and results from multiple imaging methods. Lancet 2009; 373: 897-910.

7. Onuma Y, Dudek D, Thuesen L, et al. Five-year clinical and functional multislice computed tomography angiographic results after coronary implantation of the fully resorbable polymeric everolimus-eluting scaffold in patients with de novo coronary artery disease: the ABSORB cohort A trial. JACC CardiovasC Interv 2013; 6: 999-1009.

8. Okamura T, Garg G, Gutierres-Chico JL, et al. In vivo evaluation of stent strut distribution patterns in the bioabsorbable everolimus-eluting device: an OCT ad hoc analysis of the revision 1.0 and revision 1.1 stent design in the $A B S O R B$ clinical trial. Eurolntervention 2010; 5: 932-8.

9. Diletti R, Farooq V, Girasis C, et al. Clinical and intravascular imaging outcomes at 1 and 2 years after implantation of absorb everolimus eluting bioresorbable vascular scaffolds in small vessels. Late lumen enlargement: does bioresorption matter with small vessel size? Insight from the ABSORB cohort B trial. Heart 2013; 99: 98-105.

10. Serruys PW, Onuma Y, Garcia-Garcia HM, et al. Dynamics of vessel wall changes following the implantation of the Absorb everolimus-eluting bioresorbable vascular scaffold: a multi- imaging modality study at 6, 12, 24 and 36 months. Eurolntervention 2014; 9: 1271-84.

11. Ishibashi Y, Onuma Y, Muramatsu T, et al. ABSORB EXTEND Investigators. Lessons learned from acute and late scaffold failures in the ABSORB EXTEND trial. Eurolntervention 2014; 10: 449-57.

12. Abizaid A, Costa JR Jr, Bartorelli AL, et al. The ABSORB EXTEND study: preliminary report of the twelve-month clinical outcomes in the first 512 patients enrolled. Eurolntervention 2014; pii: 20130827-06. [Epub ahead of print].

13. Serruys PW, Chevalier B, Dudek D, et al. A bioresorbable everolimus-eluting scaffold versus a metallic everolimus-eluting stent for ischaemic heart disease caused by de-novo native coronary artery lesions (ABSORB II): an interim 1-year analysis of clinical and procedural secondary outcomes from a randomised controlled trial. Lancet 2014 pii: S0140-6736(14)61455-0. doi: 10.1016/S0140-6736(14)61455-0. [Epub ahead of print].

14. Capodanno D1, Gori T, Nef H, et al. Percutaneous coronary intervention with everolimus-eluting bioresorbable vascular scaffolds in routine clinical practice: early and midterm outcomes from the European multicentre GHOST-EU registry. Eurolntervention 2014 pii: 20140707-06. doi: 10.4244/EIJY14M07_11. [Epub ahead of print].

15. Diletti R, Karanasos A, Muramatsu T, et al. Everolimus-eluting bioresorbable vascular scaffolds for treatment of patients presenting with ST- segment elevation myocardial infarction: BVS STEMI first study. Eur Heart J 2014; 35: 777-86.

16. Kocka V, Maly M, Tousek P, et al. Bioresorbable vascular scaffolds in acute ST-segment elevation myocardial infarction: a prospective multicentre study 'Prague 19'. Eur Heart J 2014; 35: 787-94.

17. Lesiak M. BVS in bifurcations - techniques, acute and long-term outcomes. Xth meeting of European Bifurcation Club, Bordeaux, 17-18 October 2014.

18. Kolandaivelu K, Swaminathan R, Gibson WJ, et al. Stent thrombogenicity early in high-risk interventional settings is driven by stent design and deployment and protected by polymer-drug coatings. Circulation 2011; 123: 1400-9. 